\title{
LA NUTRITION MINÉRALE DE L'ÉPICÉA EN HAUTE-ARDÈCHE : LIAISON AVEC LA PRODUCTION
}

\author{
F. LE TACON, H. OSWALD et R. TOMASSONE
}

avec la collaboration technique de Maryse Bitsch, D. Boucharo, Simone Desueunfs, A. Divoux, Françoise Georges, F. Michaux, Danielle Salmon et A. Tisserand

Station de Recherches sur les Sols forestiers et la Fertilisation.

Station de Sylviculture et de Production,

Centre national de Recherches forestières, 54 - Nancy

Laboratoire de Biométrie,

Centre national de Recherches zootechniques, 78 -Jouy-en-Josas

Institut national de la Recherche agronomique

\section{RESUME}

La présente étude concerne la nutrition minérale de l'épicéa (Picea abies L.) sur les hauts plateaux de l'Ardèche et de la Haute-Loire - au-dessus de $1000 \mathrm{~m}$ d'altitude - dans le Massif Central. Elle fait suite à une étude des sols et à une étude de la production en fonction de certaines caractéristiques du milieu (altitude, roche mère, etc....).

Le jugement de la nutrition en valeur absolue (analyse foliaire) a montré qu'un seul élément, l'azote, était déficitaire dans tous les peuplements, doù l'intérêt de la mise en place d'essais de fertilisation.

Différentes méthodes d'analyses statistiques (analyse canonique, régression multiple progressive, régression orthogonale et analyse des correspondances) ont permis d'étudier la nutrition sur les trois roches mères, les liaisons entre altitude et nutrition, production et nutrition. caractéristiques du sol et nutrition.

Les différents résultats mettent en évidence l'importance du phénomène de concentration en éléments minéraux (notamment pour l'azote) par diminution de la biomasse, et ses conséquences sur linterprétation des analyses foliaires.

\section{INTRODUCTION}

L'un d'entre nous (OswaLD, 1969) dans un précédent article a étudié les potentialités de l'Epicéa sur les hauts plateaux de l'Ardèche et de la Haute-Loire au-dessus de $1.000 \mathrm{~m}$ d'altitude dans le Massif Central. L'accroissement moyen à 75 ans exprimé en $\mathrm{m}^{3}$ par hectare et par an y varie de 1,3 à $12,2 \mathrm{~m}^{3 .}$. 
Ces variations de production ont été étudiées en fonction de certains facteurs du milieu : altitude, roche mère, vent, exposition et pente. Les variations daltitude expliquent à elles seules $87 \%$ de la variation de production. L'action de l'altitude est extrêmement complexe. Elle agit d'une part par modifications des conditions climatiques (diminution de la période de végétation, abaissement de la température moyenne, diminution de l'amplitude thermique annuelle, augmentation de la vitesse du vent, etc...) et d'autre part par modifications des conditions de sols. Ces dernières ont également été analysées dans un précédent article (Le TACON, OSWALD, 1969) et nous n'y reviendrons pas. Les modifications des conditions de sols doivent se répereuter sur les conditions de nutrition et par là même sur la production.

Duchaufour (1957) a montré que dans les sols d'altitude à moder pseudo alpin, malgré le $\mathrm{C} / \mathrm{N}$ bas, les jeunes plantations d'épicéa souffraient de déficience en azote. Bonneau (1967) a montré, dans des sols analogues des Vosges, que la libération d'azote minéral in situ est faible et que la nitrification est presque nulle à haute altitude, alors qu'à altitude plus basse la nitrification est forte. Par des expériences en incubation ce même auteur a démontré que des apports de calcium pouvaient améliorer la production azotée en particulier nitrique. De même, dans le Massif Central, il existe une variation systématique de la production azotée en fonction de l'altitude (Bonneau, 1967). Sur jeunes plantations d'épicéa, une amélioration de la croissance peut être obtenue par apport d'engrais phosphocalco-potassiques, malgré la richesse des sols en phosphore (BONNEAU, 1965). Cet auteur émet d'ailleurs l'hypothèse qu'une fertilisation calco-potassique pourrait améliorer la croissance et la nutrition en phosphore par minéralisation du phosphore bloqué sous forme organique.

Dans I'Erzgebirge en Allemagne, FIEDLER et NEBE (1969) ont montré que la nutrition des peuplements âgés d'épicéa situés sur les stations sommitales à sols pauvres ne différait pas de celle des peuplements à meilleure croissance situés à moyenne altitude.

Nous avons cherché à savoir si, dans l'est du Massif Central, des problèmes analogues pouvaient se rencontrer sur peuplements adultes et se répereuter sur la production.

\section{I. - METHODES DE TRAVAIL.}

\section{1. - Echantillonnage}

Nous n'insisterons pas sur les conditions de milieu qui ont été décrites en détail dans l'étude précédente (Oswald, 1969) et à laquelle on pourra se reporter. Nous avons utilisé pour notre échantillonnage les 33 placettes temporaires qui ont servi à l'étude des potentialités de l'épicéa. Rappelons que ces placettes s'étagent de 1075 à $1615 \mathrm{~m}$ et se répartissent sur trois roches mères principales :

$\mathrm{G}$ - des roches mères cristallines: granites et granites porphyroïdes, granites gneissiques, gneiss et micaschistes;

$\mathrm{B}$ - des roches mères basaltiques : basaltes, scories basaltiques ;

$\mathrm{P}$ - des roches mères volcaniques acides: phonolites, trachytes et andésites.

Dans chaque placette temporaire, nous avons choisi cinq arbres dominants. Sur chacun de ces arbres, nous avons prélevé en novembre 1968 des aiguilles de l'année dans le tiers superieur de la cime. Dans chaque placette, nous avons également décrit le sol et prélevé un échantillon par horizon. 


\section{2. - Méthodes d'analyses}

Sur chacun des prélèvements foliaires, nous avons effectué une analyse totale des éléments suivants: $\mathrm{N}, \mathrm{P}_{2} \mathrm{O}_{5}, \mathrm{~K}, \mathrm{Ca}, \mathrm{Mg}, \mathrm{Fe}$, et $\mathrm{Mn}$ après séchage à l'étuve à $65^{\circ}$ pendant $24 \mathrm{~h}$, broyage et attaque à l'acide sulfurique et à l'eau oxygénée. Les dosages ont été effectués par les méthodes habituelles.

L'azote a été dosé par distillation au micro-kjeldahl Parnass-Wagner, le phosphore par colorimétrie au molybdate d'ammonium avec réduction au chlorure stanneux. Le potassium et le calcium ont été dosés par spectrophotométrie de flamme, le magnésium par colorimétrie au jaune titane, le fer par colorimétric au sulfocyanure d'ammonium; enfin le manganèse a été dosé par colorimétrie après oxydation de l'ion $\mathrm{Mn}++$ en $\mathrm{Mn}_{4}-$ par le périodate de potassium. Sur les échantillons de sol, nous avons déterminé le carbone total par la méthode Anne, lazote total par la méthode Anstett, les éléments échangeables et la capacité d’échange par percolation à l'acétate d’ammonium, le phosphore assimilable par extraction à $\mathrm{H}_{2} \mathrm{SO}_{4}+\mathrm{NaOH}$ et le $\mathrm{pH}$ électrométriquement dans l'eau. La granulométrie a été effectuée par densimétrie (méthode Mériaux).

\section{3. - Méthodes dinterprétation}

Nous allons en premier lieu essayer de déterminer dans quelle mesure les placettes diffèrent entre elles, et quels sont les éléments qui les différencient le mieux. Par analyse de la variance à un facteur contrólé, nous testons l'hypothèse d'égalité des moyennes pour l'ensemble des placettes.

Nous chercherons ensuite à quantifier les liaisons existant entre les divers éléments en calculant les coefficients de corrélation inter et intra-peuplement. Pour différencier la nutrition sur les trois principales roches mères, nous comparerons tout dabord les moyennes des différents éléments toujours par analyse de la variance. Puis, nous utiliserons l'analyse canonique pour rechercher les combinaisons d'éléments qui nous donnent la discrimination maximale entre les roches mères.

Enfin, nous allons essayer de relier les caractéristiques de nutrition à laltitude, à la production et aux caractéristiques du sol. Pour relier la nutrition à la production et aux caractéristiques du sol, nous utilisons l'analyse par régression multiple (TomassoNE, 1967). Enfin, nous avons effectué une analyse de correspondances (BeNzícri, 1969) sur l'ensemble des caractéristiques du sol.

\section{II. - ETUDES DE LA NUTRITION PROPREMENT DITE}

\section{1. - Jugement de la nutrition en valeur absolue.}

Nous commençons à connaitre les valeurs optimales des niveaux de nutrition en divers éléments des résineux.

Le tableau 1 nous montre que le niveau de nutrition en azote est dans l'ensemble très bas. La moyenne des teneurs en azote des aiguilles est en effet de 1,26\% alors que la valeur optimale se situe aux environs de 1,6 à $1,8 \%$. Le maximum observé $(1,47 \%)$ est encore loin de loptimum et le minimum descend au-dessous de $1 \%$ $(0,98 \%)$.

Le niveau de nutrition en phosphore est par contre à l'optimum. La moyenne observée en $\mathrm{P}_{2} \mathrm{O}_{5}$ est de $0,41 \%$ alors que l'on admet que l'optimum se situe entre 0,36 et $0,40 \%$. Même les valeurs les plus faibles que nous avons observées sont au niveau de loptimum $(0,34 \%)$.

Le niveau de nutrition en potassium est également très élevé. L'optimum admis correspond à une teneur en potassium des aiguilles de $0,50 \%$. Or, la moyenne de l'ensemble des placettes est de $0,69 \%$. Comme pour le phosphore même les valeurs les plus basses sont au niveau optimal. 
TABLEAU 1 - TABLE I

Caractéristiques des placettes et résultats de ranalyse foliaire (valeurs moyennes de 5 arbres dominants)

Characteristics of semi-permanent sample plots and results of foliar analysis (mean of five dominant trees)

\begin{tabular}{|c|c|c|c|c|c|c|c|c|c|c|c|c|c|c|c|}
\hline \multirow{2}{*}{$\begin{array}{l}\frac{0}{\tilde{U}} \\
\frac{\tilde{H}}{a} \\
z \\
z\end{array}$} & \multirow{2}{*}{ 祃 } & \multirow{2}{*}{ 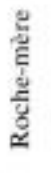 } & \multirow{2}{*}{ 㼵 } & \multirow{2}{*}{ 总兽 } & \multirow{2}{*}{ 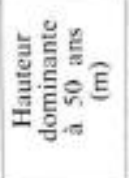 } & \multirow{2}{*}{ 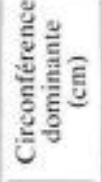 } & \multirow{2}{*}{ 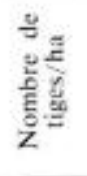 } & \multirow{2}{*}{ 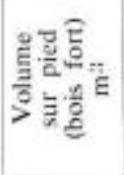 } & \multicolumn{7}{|c|}{ Analyses foliaires } \\
\hline & & & & & & & & & $\underset{(\%)}{N}$ & $\underset{(\mathrm{S}) \mathrm{O}_{5}}{\mathrm{P}_{4} \mathrm{O}_{5}}$ & $\begin{array}{c}\mathrm{K} \\
(\%)\end{array}$ & $\begin{array}{l}\mathrm{Ca} \\
(\mathrm{E})\end{array}$ & $\begin{array}{c}\mathrm{Mg} \\
\tilde{g}\end{array}$ & $\begin{array}{c}\mathrm{Mn} \\
(\because,)\end{array}$ & $\begin{array}{c}\mathrm{Fe} \\
(\%)\end{array}$ \\
\hline 0.701 & 1.170 & G & 73 & 28,9 & 23,6 & 147 & 528 & 679 & 1,25 & 0,36 & 0.67 & 0,54 & 0.11 & 0.20 & 0,02 \\
\hline 0.702 & 1.345 & G & 75 & 27,0 & 20,2 & 137 & 650 & 662 & 1.31 & 0,43 & 0.77 & 0,64 & 0.13 & 0,40 & 0,05 \\
\hline 0.703 & 1.250 & $B$ & 87 & 21,3 & 12,6 & 115 & 1.020 & 565 & 1,23 & 0,41 & 0,84 & 0,75 & 0,16 & 0.44 & 0,02 \\
\hline 0.704 & 1.130 & G & 76 & 29.4 & 24,1 & 139 & 557 & 623 & 1,20 & 0,35 & 0,75 & 0,43 & 0,11 & 0,40 & 0,04 \\
\hline 0.705 & 1.440 & $\mathrm{P}$ & 94 & 21.6 & 12,8 & 139 & 459 & 471 & 1.28 & 0,40 & 0,47 & 0,57 & 0,08 & 1,00 & 0,06 \\
\hline 0.706 & 1.427 & G & 84 & 23,2 & 15,7 & 135 & 615 & 608 & 1,27 & 0,46 & 0.67 & 0,47 & 0.15 & 0,94 & 0.05 \\
\hline 0.707 & 1.380 & G & 83 & 28,2 & 21.1 & 144 & 453 & 587 & 1,34 & 0.42 & 0,64 & 0,68 & 0,14 & 1,32 & 0,01 \\
\hline 0.708 & 1.145 & G & 79 & 27,3 & 21,1 & 137 & 429 & 538 & 1.19 & 0,42 & 0,62 & 0,45 & 0,16 & 0,94 & 0,02 \\
\hline 0.709 & 1.338 & $\mathrm{G}$ & 80 & 28,7 & 20,7 & 169 & 321 & 639 & 1,19 & 0,38 & 0,66 & 0,46 & 0,20 & 1,14 & 0.05 \\
\hline 0.710 & 1.430 & G & 64 & 22,3 & 18,8 & 121 & 449 & 454 & 1,24 & 0.44 & 0.74 & 0,53 & 0.10 & 0,96 & 0,06 \\
\hline 0.711 & 1.320 & $\bar{G}$ & 77 & 25,9 & 20,4 & 130 & 408 & 448 & 1,10 & 0,34 & 0,47 & 0.49 & 0,13 & 1,05 & 0,05 \\
\hline 0.712 & 1.422 & G & 61 & 20,8 & 17,1 & 114 & 497 & 392 & 1.27 & 0.39 & 0,69 & 0.75 & 0,17 & 1,26 & 0.07 \\
\hline 0.713 & $\begin{array}{l}1.415 \\
1.450\end{array}$ & G & & $\begin{array}{l}21,4 \\
176\end{array}$ & $\begin{array}{l}17.5 \\
16.0\end{array}$ & 118 & $\begin{array}{l}508 \\
669\end{array}$ & 441 & $\begin{array}{r}1,39 \\
1,36\end{array}$ & 0,39 & 0,67 & 0,58 & 0,11 & 0,90 & 0,04 \\
\hline & $\begin{array}{l}1.450 \\
1.455\end{array}$ & $\underset{\mathrm{G}}{\mathrm{G}}$ & $\begin{array}{l}63 \\
78\end{array}$ & $\begin{array}{l}17,6 \\
19,2\end{array}$ & $\begin{array}{l}16,9 \\
11,7\end{array}$ & $\begin{array}{l}108 \\
114\end{array}$ & $\begin{array}{r}669 \\
1.222\end{array}$ & $\begin{array}{l}351 \\
532\end{array}$ & $\begin{array}{l}1,36 \\
1,27\end{array}$ & $\begin{array}{l}0,39 \\
0,36\end{array}$ & $\begin{array}{l}0,62 \\
0.71\end{array}$ & $\begin{array}{l}0,67 \\
0.65\end{array}$ & 0,14 & $\begin{array}{l}1,35 \\
0,94\end{array}$ & 0,05 \\
\hline $\begin{array}{l}0.715 \\
0.716\end{array}$ & $\begin{array}{l}1.455 \\
1.075\end{array}$ & $\mathrm{G}$ & $\begin{array}{l}78 \\
91\end{array}$ & 31,9 & 18,8 & $\begin{array}{l}114 \\
129\end{array}$ & 504 & 496 & $\begin{array}{l}1.27 \\
1.20\end{array}$ & 0,37 & 0.52 & 0.54 & 0,18 & 1,39 & $\begin{array}{l}0,06 \\
0,13\end{array}$ \\
\hline 0.717 & 1.490 & $\mathbf{P}$ & 84 & 17,6 & 10,2 & 113 & 532 & 304 & 1.32 & 0,46 & 0.64 & 0,50 & 0,09 & 0,57 & 0,05 \\
\hline 4.315 & 1.515 & $\mathbf{P}$ & 72 & 17,3 & 12,6 & 101 & 1.232 & 424 & 1,21 & 0.40 & 0,87 & 0,36 & 0,08 & 0,74 & 0,07 \\
\hline 4.316 & 1.282 & B & 86 & 30,2 & 19.8 & 146 & 875 & 938 & 1,21 & 0,39 & 0,66 & 0,77 & 0,25 & 0,23 & 0,11 \\
\hline 4.317 & 1.265 & B & 81 & 27,9 & 19.0 & 168 & 451 & 694 & 1,04 & 0,53 & 0.78 & 0,82 & 0.15 & 0,41 & 0,09 \\
\hline 4.318 & 1.400 & $\mathrm{P}$ & 81 & 21,6 & 13,6 & 129 & 555 & $\begin{array}{l}493 \\
389\end{array}$ & 1,24 & 0.44 & 0,82 & 0,52 & 0,09 & 0.55 & 0,08 \\
\hline $\begin{array}{l}4.319 \\
4320\end{array}$ & & $\begin{array}{l}\mathrm{P} \\
\mathrm{P}\end{array}$ & 85 & 18,6 & 11,8 & $\begin{array}{l}115 \\
133\end{array}$ & 604 & $\begin{array}{l}389 \\
454\end{array}$ & $\begin{array}{l}124 \\
1.26\end{array}$ & $\begin{array}{l}0,40 \\
0,42\end{array}$ & $\begin{array}{l}0,71 \\
0,57\end{array}$ & $\begin{array}{l}0.42 \\
0.75\end{array}$ & $\begin{array}{l}0,10 \\
0.26\end{array}$ & $\begin{array}{l}0.68 \\
0.78\end{array}$ & 0,10 \\
\hline $\begin{array}{l}4.320 \\
4.321\end{array}$ & $\begin{array}{l}1.372 \\
1.265\end{array}$ & $\begin{array}{l}\mathrm{P} \\
\mathrm{B}\end{array}$ & $\begin{array}{l}82 \\
47\end{array}$ & 22,7 & 13,8 & $\begin{array}{l}133 \\
127\end{array}$ & $\begin{array}{l}460 \\
907\end{array}$ & $\begin{array}{l}454 \\
360\end{array}$ & & $\begin{array}{l}0.42 \\
0.43\end{array}$ & $\begin{array}{l}0.57 \\
0.87\end{array}$ & $\begin{array}{l}0.75 \\
0.89\end{array}$ & $\begin{array}{l}0,26 \\
0.14\end{array}$ & $\begin{array}{l}0,78 \\
0,30\end{array}$ & 0,07 \\
\hline 4.322 & 1.615 & $\mathrm{P}$ & $\begin{array}{l}42 \\
84\end{array}$ & $\begin{array}{l}19,5 \\
11,3\end{array}$ & $\overline{5,7}$ & $\begin{array}{r}127 \\
98\end{array}$ & $\begin{array}{r}907 \\
1.549\end{array}$ & $\begin{array}{l}560 \\
272\end{array}$ & $\begin{array}{l}0,98 \\
1.27\end{array}$ & $\begin{array}{l}0,43 \\
0,41\end{array}$ & $\begin{array}{l}0.87 \\
0.65\end{array}$ & $\begin{array}{l}0,89 \\
0,29\end{array}$ & 0,06 & $\begin{array}{l}0,30 \\
0.55\end{array}$ & 0,08 \\
\hline 4.323 & 1.535 & $\mathrm{P}$ & 87 & 15,5 & 8,1 & 137 & 672 & 339 & 1,33 & 0,40 & 0,81 & 0,33 & 0,07 & 0.66 & $\begin{array}{l}0,07 \\
0,09\end{array}$ \\
\hline 4.324 & 1.570 & P & 82 & 12,5 & 6,7 & 105 & 1.119 & 253 & 1,47 & 0,43 & 0.88 & 0,36 & 0,07 & 0.25 & $\begin{array}{l}0,09 \\
0,07\end{array}$ \\
\hline 4.325 & 1.210 & B & 78 & 26,5 & 19,3 & 138 & 591 & 564 & 1,19 & 0,49 & 0,48 & 0,48 & 0,17 & 0,32 & 0,05 \\
\hline 4.326 & 1.300 & B & 82 & 29,1 & 19.6 & 138 & 840 & 889 & 1.29 & 0.46 & 0,78 & 0,54 & 0,10 & 0,36 & 0,03 \\
\hline 4.327 & 1.375 & B & 78 & 26,2 & 16,9 & 122 & 1.063 & 845 & 1.35 & 0.35 & 0.75 & 0,60 & 0,12 & 0,48 & 0,04 \\
\hline 4,328 & 1.330 & B & 81 & 27,2 & 18,0 & 119 & 834 & 750 & 1.38 & 0,41 & 0.74 & 0.60 & 0.14 & 0.18 & 0.08 \\
\hline 4.329 & 1.390 & B & 50 & 20.9 & 20,9 & 118 & 1.313 & 598 & 1,28 & 0,40 & 0,48 & 0.57 & 0,16 & 0,17 & 0,01 \\
\hline 4.330 & 1.238 & B & 36 & 17.5 & - & 100 & 1.742 & 428 & 1,27 & 0,51 & 0,99 & 1,01 & 0,09 & 0,25 & 0,06 \\
\hline
\end{tabular}


Le niveau moyen de nutrition en calcium est de $0,58 \%$ et certaines placettes descendent à un niveau inférieur à $0,35 \%$. Mais on ne peut parler de déficiences. II est d'ailleurs difficile de parler de seuil de carence ou de valeur optimale pour le calcium. En général, le seuil de carence proprement dit n'est jamais atteint quelles que soient les conditions de sols. Il y a toujours suffisamment de calcium pour assurer l'approvisionnement du végétal en cet élément. Mais le calcium a de nombreux effets indirects: la minéralisation de la matière organique par exemple dépend beaucoup de la teneur en calcium du sol. Il peut donc y avoir suffisamment de calcium dans le sol pour approvisionner directement le végétal, mais insuffisamment pour assurer une nutrition convenable en autres éléments. On peut done penser que des teneurs en calcium inférieures à $0,40 \%$ dans les aiguilles si elles sont intrinsèquement suffisantes, peuvent néanmoins être considérées comme faibles.

Dans l'ensemble, le niveau de nutrition en magnésium est satisfaisant: moyenne $0,12 \%$, optimum $0,10 \%$. Parfois, néanmoins, le niveau de nutrition en cet élément devient faible (minimum observé $0,066 \%$ ). Enfin, le niveau de nutrition en manganèse est toujours élevé.

TABLEAU 2 - TABLE 2

Analyse de la variance pour chaque elément minéral ef pour lensemble des placettes Analysis of variance for each mineral element and for all plots

\begin{tabular}{|c|c|c|c|c|c|}
\hline \multirow{2}{*}{ Caractères } & \multirow{2}{*}{$\begin{array}{l}\text { Moyenne } \\
\text { observéc }\end{array}$} & \multicolumn{2}{|c|}{$\begin{array}{c}\text { Valeurs extrêmes } \\
\text { observecs }\end{array}$} & \multirow{2}{*}{$\begin{array}{l}\text { Test } F \\
\text { ddl }=32 \\
\text { et } 132\end{array}$} & \multirow{2}{*}{ Significution } \\
\hline & & Max. & Min. & & \\
\hline $\operatorname{Mn} \quad \ldots, \ldots$. & 0,69 & 1,38 & 0,16 & 15,52 & $x x$ \\
\hline $\mathbf{K} \quad \mathrm{s}_{0} \quad \ldots \ldots \ldots$ & 0,69 & 0,47 & 0,99 & 7,91 & $x x$ \\
\hline $\mathrm{Ca} \% \ldots$ & 0,58 & 0,33 & 1.01 & 7,06 & $x x$ \\
\hline $\mathrm{Fe} \%, \ldots, \ldots$ & 0.06 & 0,13 & 0,01 & 4,29 & $x x$ \\
\hline $\mathbf{P}_{2} \mathbf{O}_{z}, S_{0} \ldots$. & 0,41 & 0,53 & 0,34 & 3,43 & $x x$ \\
\hline $\mathrm{Mg} \%$ & 0,12 & 0,20 & 0,06 & 3,07 & $x x$ \\
\hline $\mathrm{N} \% \ldots \ldots$ & 1,26 & 1,47 & 0,98 & 2,88 & $x x$ \\
\hline
\end{tabular}

\section{2. - Analyse de la variance à un facteur contrôlé (ensemble des placettes)}

Les résultats de cette analyse sont consignés dans le tableau 2. Tous les tests sont significatifs au seuil de $1 \%$. Par conséquent, les peuplements diffèrent globalement par tous les éléments dosés. Il est intéressant de commenter l'ordre de classement en fonction des valeurs de F. Nous constatons que c'est le manganèse qui discrimine le plus les peuplements. Viennent ensuite le potassium et le calcium avec des rapports F encore élevés. L'élément qui discrimine le moins l'ensemble des peuplements est l'azote. Ce résultat concernant l'azote est assez inattendu. En effet, nous pensions qu'en raison des fortes variations d'altitude d'une part, et des importantes variations de disponibilité du sol en éléments échangeables d'autre part, que la nutrition azotée serait un facteur de discrimination important. Or, ce n'est pas le cas bien que le test $\mathrm{F}$ soit significatif. 
Une fois encore, nous constatons que c'est le manganèse qui présente le pouvoir discriminant le plus important. Nous reviendrons dans la suite de ce mémoire sur le comportement du manganèse.

\section{3. - Corrélations entre éléments}

Nous avons calculé la matrice des coefficients de corrélation intra-peuplement et inter-pcuplement (tableau 3).

\section{TABLEAU 3 - TABLE 3}

Matrices des coefficients de correlation fotaux significatits (coefficients intra et inter-peuplement) Matrix of significative total correlation coefficients (inter and intra correlation coefficients)

\begin{tabular}{|c|c|c|c|c|c|c|c|c|}
\hline 1 & $\mathrm{~N}$ & $\circ$ & & & & & & \\
\hline 2 & $P$ & $+0,43$ & 0 & & & & & \\
\hline 3 & K & 0 & $+0,37$ & a & & & & \\
\hline 4 & $\mathrm{Ca}$ & $a$ & 0 & 0 & 0 & & & \\
\hline 5 & $\mathrm{Mg}$ & 0 & 0 & 。 & o & o & & \\
\hline 6 & $\mathrm{Mn}$ & 0 & . & $-0,21$ & $+0,53$ & 0 & 0 & \\
\hline 7 & $\mathrm{Fe}$ & 0 & 0 & 0 & 0 & 0 & $\circ$ & 0 \\
\hline & & $\mathrm{N}$ & P & K & $\mathrm{Ca}$ & $\mathrm{Mg}$ & $\mathrm{Mn}$ & $\mathrm{Fe}$ \\
\hline & & 1 & 2 & 3 & 4 & 5 & 6 & 7 \\
\hline
\end{tabular}

matrice intra $(r$ au seuil de $5 \%=0,194)$

( $r$ au seuil de $1 \%=0,254)$

\begin{tabular}{|c|c|c|c|c|c|c|c|c|}
\hline 1 & $N$ & 0 & & & & & & \\
\hline 2 & $\mathbf{P}$ & a & 0 & & & & & \\
\hline 3 & K & a & $\circ$ & 0 & & & & \\
\hline 4 & $\mathrm{Ca}$ & 0 & 0 & 0 & 0 & & & \\
\hline 5 & $\mathrm{Mg}$ & $-0,35$ & 0 & $-0,41$ & $+0,36$ & $\circ$ & & \\
\hline 6 & $\mathrm{Mn}$ & 0 & $-0,36$ & $-0,42$ & o & 0 & D & \\
\hline \multirow[t]{3}{*}{7} & $\mathrm{Fe}$ & o & a & 0 & D & 0 & 0 & o \\
\hline & & $\mathrm{N}$ & P & $\mathrm{K}$ & $\mathrm{Ca}$ & $\mathrm{Mg}$ & Mn & $\mathrm{Fc}$ \\
\hline & & I & 2 & 3 & 4 & 5 & 6 & 7 \\
\hline
\end{tabular}

matrice inter $(r$ au seuil de $5 \%=0,349)$

( $r$ au seuil de $1 \%=0,448$ )

Rappelons que le coefficient de corrélation intra-peuplement entre deux caractères quantifie leur liaison à l'intérieur d'un peuplement. Ces liaisons peuvent avoir de multiples causes. Il traduit la liaison * moyenne * entre deux caractères après élimination de la variabilité entre les traitements et nous avons admis dans un précédent article (LE TACON-Millier, 1970) que ces liaisons pouvaient en particulier traduire des rôles complémentaires dans certains processus physiologiques. A l'opposé, les coefficients de 
corrélation inter-peuplement traduisent laction des facteurs de milieu, sans cependant qu'ils soient \& épurés * des variations intra-peuplement.

Les résultats que nous avons obtenus ne sont malheureusement pas clairs. Considérons les coefficients de corrélation intra-peuplement: il existe trois séries de liaisont très significatyives: N-P, P-K, et Ca-Mn, toute positives. Nous retrouvons la liaison positive calcium-manganèse que nous avions déjà signalée (LE TACON-MiLlıER, 1970).

Les coefficients de corrélation inter-peuplement que nous obtenons, sont pratiquement ininterprétables. Sur cinq, quatre sont négatifs. Seule la liaison entre calcium et magnésium est positive. La matrice des coefficients inter-peuplement n'est intéressante que par ce qu'elle ne fait pas apparaitre. Il n'existe pas de liaison entre nutrition azotée et nutrition en potassium et calcium. Or, nous aurions pu nous attendre à une nette amélioration de la nutrition azotée lorsque le niveau de disponibilité en cations du sol augmente. Ce n'est absolument pas le cas et nous trouvons même une liaison négative entre $\mathrm{N}$ et $\mathrm{Mg}$. D'autre part, il n'existe pas de liaison négative significative entre calcium et manganèse comme nous l'avions observé dans l'est de la France, sur sol à forte disponibilité en calcium. A priori, nous pouvons essayer d'interpréter ce fait de la manière suivante : nous pouvons supposer que lorsque la disponibilité du sol en calcium est faible ou peu élevée, l'absorption du manganèse se fait sans difficulté. Nous ne mettons donc en évidence aucune liaison négative entre calcium et manganèse. En fait, cette explication nous le verrons par la suite n'est pas satisfaisante. Un phénomène très important est venu complètement perturber les teneurs en éléments minéraux des aiguilles, et rend les interprétations habituelles impossibles. De plus, l'existence de discontinuité entre les rozhes-mères, mises en évidence par les tests $F$, en particulier pour le manganèse, fait perdre une grande partie de leur validité statistique aux corrélations inter-peuplement.

\section{III. - MISE EN EVIDENCE DES DIFFERENCES DE NUTRITION ENTRE LES TROIS TYPES DE ROCHES MERES}

\section{1. - Analyse de la variance}

Nous avons effectué une analyse de la variance à un facteur contrôlé pour essayer de différencier la nutrition sur les trois roches-mères. Il existe quatorze points d'observations sur granite, dix sur basalte et neuf sur phonolite.

Les résultats sont consignés dans le tableau 4. Seuls quatre éléments diffèrent significativement entre les peuplements situés sur les trois roches-mères. Ce sont dans l'ordre: le manganèse, le magnésium, le calcium et le phosphore.

Sur les trois types de roche-mère, les peuplements diffèrent tous entre eux par leur niveau de nutrition en manganèse. Le niveau le plus faible est observé sur basalte et le plus élevé sur granite. En ce qui concerne le magnésium, seuls les peuplements situés sur phonolite se distinguent des deux autres par un niveau plus faible. II n'y a pas de différence significative entre les peuplements situés sur basalte et ceux situés sur granite. Par contre, le niveau de nutrition en calcium diffère pour les trois roches-mères deux à deux. Le niveau le plus faible est observé sur phonolite (moyenne $0,45 \%$ ) et 
TABLEAU 4 - TABLE 4

Analyse de la variance à un facteur contrôlé : comparaison de la nutrition sur les trois roches-mères Analysis of variance : comparison of nutrition on the three parent rocks

\begin{tabular}{|c|c|c|c|c|c|c|c|}
\hline & $\mathrm{N} \%$ & $\mathrm{P}_{2} \mathrm{O}_{5} \%$ & $\mathrm{~K} \%$ & $\mathrm{Ca} \%$ & $\mathrm{Mg} \%$ & Mn Fe & $\mathrm{Fe} \%$ \\
\hline Phonolites ... & 1,298 & 0,416 & 0.711 & 0,45 & 0,08 & 0,64 & 0,07 \\
\hline Granites.... & 1,261 & 0,392 & 0,655 & 0,563 & 0.14 & 0,99 & 0,05 \\
\hline Basaltes ....... & 1,225 & 0,436 & 0.735 & 0,703 & 0,13 & 0,31 & 0,05 \\
\hline $\mathbf{F} \ldots \ldots \ldots$ & $1,41 \mathrm{NS}$ & $3,435^{\circ}$ & $1,227 \mathrm{NS}$ & $7,84=28$ & $8,708^{\circ \Rightarrow}$ & $24,538^{\circ 6}$ & $2,548 \mathrm{NS}$ \\
\hline
\end{tabular}

le plus élevé sur basalte (moyenne $0,70 \%$ ). Enfin, le niveau de nutrition en phosphore ne diffère significativement qu'entre les peuplements situés sur granite (moyenne $0,39 \%$ ) et ceux situés sur basalte (moyenne $0,44 \%$ ).

\section{2. - Analyse canonique}

Nous avons effectué deux analyses canoniques différentes. Nous avons tout d'abord effectué une analyse canonique sans différencier au départ les peuplements situés sur les trois roches-mères. Puis, nous avons effectué une seconde analyse en groupant les peuplements par roche-mère. Nous calculons dans les deux cas, les composantes canoniques qui ont la propriété de fournir la meilleure discrimination, à partir de la matrice des coefficients de corrélation totaux déterminés d'une part entre les populations et d'autre part, à l'intérieur des populations. Avec $p$ variables et $k$ populations, il existe un nombre de variables canoniques égal, au minimum à $(p, k-1)$. Pour pouvoir les interpréter facilement, il est nécessaire de travailler sur les variables initiales réduites.

\subsection{Analyse canonique effectuée sans différencier les roches-mères au départ.}

Dans cette analyse, les populations sont constituées par les arbres. II existe donc 33 populations constituées chacune par cinq arbres. Six composantes canoniques discriminent significativement l'ensemble de ces 33 populations. Nous avons calculé les valeurs numériques relatives au point moyen des 33 peuplements et reporté sur des graphiques les combinaisons relatives à ces six composantes prises deux à deux.

En utilisant les axes des composantes $n^{\circ} 1$ et 6 , nous avons obtenu l'image permettant de discriminer au mieux les trois roches-mères (fig. $1 \mathrm{~A}$ ).

La première composante qui fait intervenir positivement le manganèse et négativement le calcium (tableau 5 A) classe parfaitement les trois roches-mères. Par contre, la seconde qui fait intervenir positivement le phosphore et négativement le fer et le magnésium est nettement moins discriminante. Elle ne permet de différencier que les phonolites des deux autres roches-mères. 


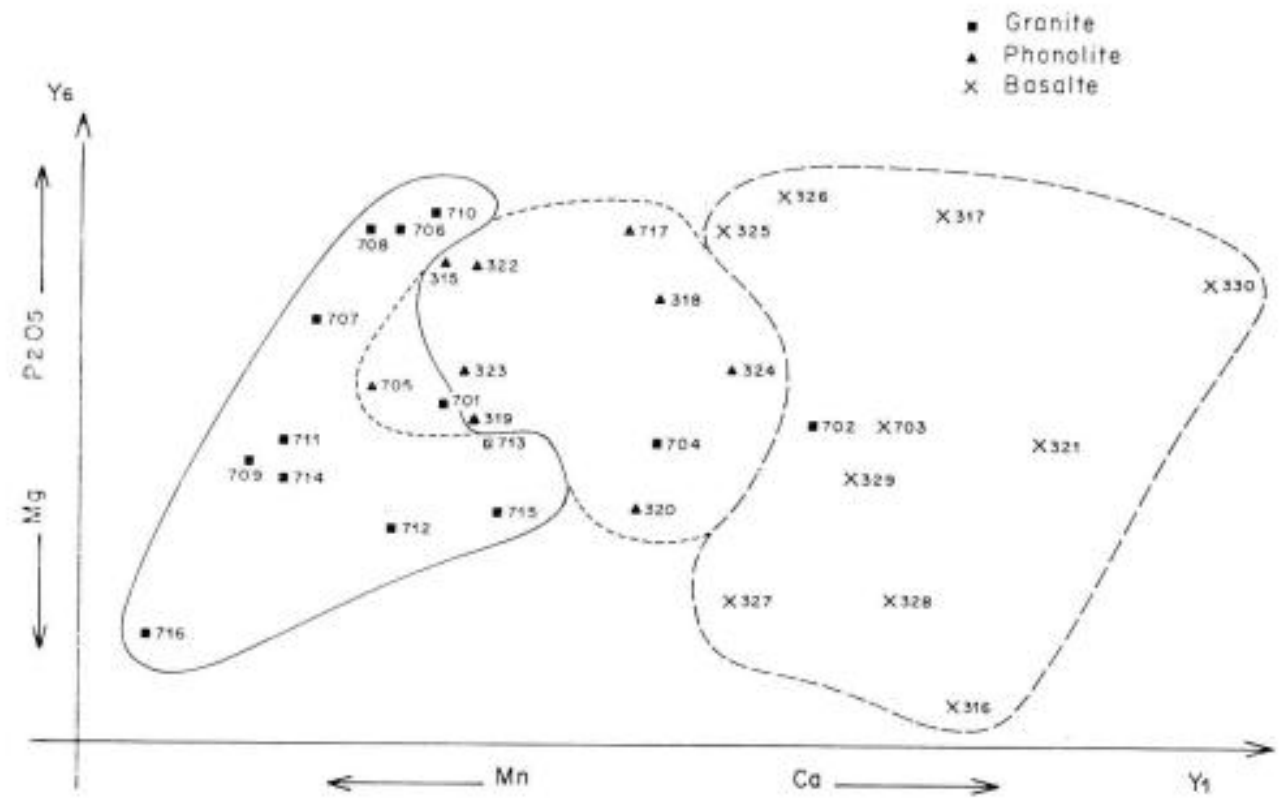

FiG. 1 A. - Représentation graphique des 33 peuplements dans le plan des deux axes canoniques $Y_{1}$ et $Y_{\text {, }}$

Fic. I A. - Graph of the 33 stands in the plane of two canonical axis: $Y_{1}$ and $Y_{6}$

\section{TABLEAU $5 \mathrm{~A}$ - TABLE $5 \mathrm{~A}$}

Analyse canonique effectuée sur 33 populations de cinq individus. Axes canoniques $Y_{1}$ et $Y_{6}$ : importance des variables initates dans chaque axe déterminée par la valeur du coefficient ${ }_{\mathrm{T}}$

$$
\begin{aligned}
(\mathrm{r} & =\mathrm{a} V \text { carré moyen de ferreur } \\
\mathrm{a}^{2} & =10.000 \text { pour chaque coeff. } \times 100)
\end{aligned}
$$

Canonical analysis with 33 populations of five samples. Canonical axis $Y_{1}$ and $Y_{n}$ : importance of each initial variable determined on each axis by the value of coefficient $r$

$$
(\mathrm{r}=\mathrm{a} \times \text { mean square of error, }
$$

\begin{tabular}{|c|c|c|c|c|c|c|c|c|}
\hline & & $\mathrm{N}$ & $\mathrm{P}_{2} \mathrm{O}_{\overline{7}}$ & K & $\mathrm{Ca}$ & $\mathrm{Mg}$ & $\mathrm{Mn}$ & $\mathrm{Fe}$ \\
\hline$Y_{1}$ & $a$ & $\begin{array}{l}+0,016 \\
+0,21\end{array}$ & $\begin{array}{r}+\quad 0,25 \\
+\quad 3,22\end{array}$ & $\begin{array}{l}+0,16 \\
+2,03\end{array}$ & $\begin{array}{l}+0,71 \\
+9,07\end{array}$ & $\begin{array}{l}-0.06 \\
-0.84\end{array}$ & $\begin{array}{l}-0,58 \\
-7,45\end{array}$ & $\begin{array}{r}-0,18 \\
-\quad 2,39\end{array}$ \\
\hline$Y_{c_{f}}$ & $\begin{array}{l}a \\
r\end{array}$ & $\begin{array}{l}-0,12 \\
-2,65\end{array}$ & $\begin{array}{r}+\quad 0,69 \\
+14,54\end{array}$ & $\begin{array}{l}+0,03 \\
+0,69\end{array}$ & $\begin{array}{l}-0,09 \\
-2,08\end{array}$ & $\begin{array}{l}-0,37 \\
-7,92\end{array}$ & $\begin{array}{l}+0,031 \\
+0,66\end{array}$ & $\begin{array}{r}-0,59 \\
-12,38\end{array}$ \\
\hline
\end{tabular}

$\mathrm{a}^{2}=10.000$ for each axis, coeff. $\times 100 \mathrm{y}$

3.22. Analyse canonique effectuée en différenciant les roches-mères au départ.

Dans cette analyse, les populations sont les peuplements de chacune des trois roches-mères. Il existe done trois populations constituées chacune par 14,9 et 10 peu- 
plements. Les caractéristiques de nutrition de chaque peuplement sont représentées par la moyenne des caractéristiques de nutrition de chacun des cinq arbres.

Les résultats sont consignés dans le tableau 5 B. Seules deux composantes sont définies pour séparer les trois roches-mères. Nous voyons que dans la première composante interviennent essentiellement le manganèse, et, à un degré moindre, le calcium avec un signe opposé, et dans la seconde le magnésium seul.

\section{TABLEAU $5 \mathrm{~B}$ - TABLE $5 \mathrm{~B}$}

Analyse canonique effectuée sur 3 populations composées de 14, 9 ef 10 peuplements. Axes canoni-

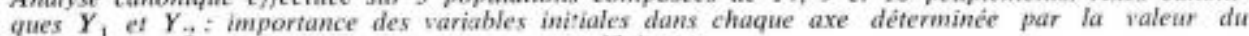
coefficient $\mathrm{r}$

$$
\begin{gathered}
\quad \mathrm{r}=\mathrm{a} \sqrt{\text { carré moyen de lerreur }} \\
\left.\mathrm{a}^{2}=10,000 \text { pour chaque axe, coeff, } \times 100\right)
\end{gathered}
$$

Canonical analysis with 3 populations of 14,9 and 10 stants. Canonical axis $Y_{1}$ and $Y_{15}$; importance of each variable determined on each axis by the value of $\mathrm{r}$ coefficient $(\mathrm{r}=\mathrm{a} \sqrt{\text { mean square of error. }}$

\begin{tabular}{|c|c|c|c|c|c|c|c|c|}
\hline & & $\mathrm{N}$ & $\mathrm{P}_{z} \mathrm{O}_{z}$ & $\mathrm{~K}$ & $\mathrm{Ca}$ & $\mathrm{Mg}_{\mathrm{g}}$ & $\mathrm{Mn}$ & $\mathrm{Fe}$ \\
\hline $\mathbf{Y}_{2}$ & a & $\begin{array}{r}-0,040 \\
-0,384\end{array}$ & $\begin{array}{l}-0,217 \\
-0,90\end{array}$ & $\begin{array}{l}+0,125 \\
+1,61\end{array}$ & $\begin{array}{l}-0,221 \\
-3,09\end{array}$ & $\begin{array}{l}-0,107 \\
-0,99\end{array}$ & $\begin{array}{l}+0,303 \\
+13,33\end{array}$ & $\begin{array}{l}-0,508 \\
-1,35\end{array}$ \\
\hline$Y_{1}$ & $\begin{array}{l}a \\
r\end{array}$ & $\begin{array}{l}-0,076 \\
-0,72\end{array}$ & $\begin{array}{l}-0,150 \\
-0,62\end{array}$ & $\begin{array}{l}+0,041 \\
+0,53\end{array}$ & $\begin{aligned} & 0.088 \\
+ & 1.20\end{aligned}$ & $\begin{aligned} & 0,691 \\
+ & 6,40\end{aligned}$ & $\begin{array}{l}-0,007 \\
-0,16\end{array}$ & $\begin{array}{l}-0,620 \\
-1,65\end{array}$ \\
\hline
\end{tabular}

$\mathrm{a}^{2}=10,000$ for each axis, coeff. $\left.\times 100\right)$

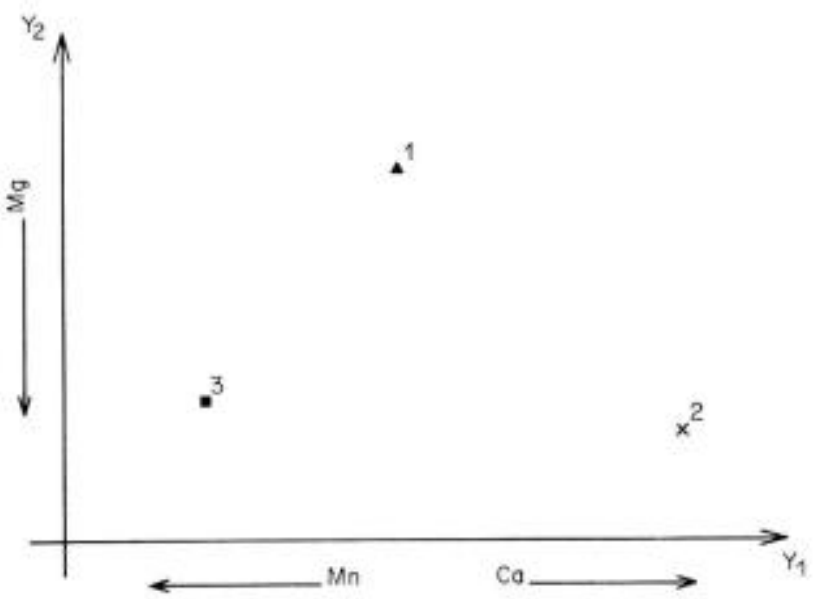

FIG. 1 B. - Représentation graphigue des trois types de peuplement dans le plan des deux axes canoniques $Y_{1}$ et $Y_{2}(I=$ phonolite: $2=$ basalte $; 3=$ granite $)$

Fio. 1 B. - Graph of the 3 types of stand in the plane of two canonical axis: $Y_{1}$ and $Y_{2}$ ( $1=$ phonolite $; 2=$ basalt $; 3=$ granite $)$ 
Nous avons calculé les valeurs numériques relatives au point moyen des trois roches-mères et porté ces résultats sur un graphique (figure $1 \mathrm{~B}$ ).

Les résultats sont très clairs et confirment lanalyse de la variance du paragraphe précédent et l'analyse canonique effectuée sur les 33 populations. On notera cependant que le phosphore qui intervenait faiblement dans les analyses précédentes n'apparaît plus ici.

Globalement nous pouvons donc dire que la nutrition des peuplements d'épicéa sur les trois roches mères principales diffère essentiellement par le niveau de nutrition en calcium, en magnésium et en manganèse. Le magnésium, deuxième variable, isole bien les phonolites par rapport aux deux autres roches.

Le niveau de nutrition en ces deux cations est le plus élevé sur basalte, ce qui se répercute sur la nutrition en manganèse dont le niveau commence à diminuer sur cette roche mère,

Par contre, comme nous l'avions déjà souligné après l'analyse de la variance effectuée sur l'ensemble des placettes (paragraphe 2.2.), la disponibilité élevée en calcium et magnésium sur basalte ne se répercute pas sur le niveau de nutrition azotée, ce qui eat le fait le plus important que nous pouvons tirer de ces différentes analyses.

\section{IV. - LIAISON ALTITUDE NUTRITION}

Nous avons calculé les coefficients de corrélaton entre les teneurs en éléments minéraux des aiguilles et l'altitude.

Trois éléments varient significativement dans le même sens que l'altitude ; ce sont par ordre de signification, le magnésium, l'azote et le calcium. Le niveau de nutrition en magnésium et calcium diminue avec l'altitude, ce qui s'explique assez facilement puisqu'à haute altitude les peuplements d'épicéa sont situés en partie sur des sols issus de phonolite et par conséquent désaturés. Au contraire les peuplements de basse altitude sont situés en partie sur des sols à taux de saturation élevé issus de basalte.

L'azote par contre présente une corrélation positive avec l'altitude ce qui est absolument contraire à tout ce à quoi nous aurions pu nous attendre. La liaison entre teneur en azote des aiguilles et altitude ne semble d'ailleurs pas linéaire (cf. fig. 2). Cependant la dispersion est telle que la liaison est assez faible : on ne peut raisonnablement parler que d'une tendance.

Pour les 33 points l'ajustement le meilleur est une droite :

$$
\begin{aligned}
\mathrm{N}=0.739+0.384 \times(\mathrm{N} & =\text { teneur en azote des aiguilles en } \% \\
\mathrm{x} & \left.=\frac{\text { altitude en } \mathrm{m}}{1000}\right)
\end{aligned}
$$

Si on élimine les deux points les plus aberrants, la meilleure estimation est obtenue en utilisant le carré de l'altitude. On obient ainsi une courbe dont l'équation est la suivante :

$$
\mathrm{N}=1,074+0,108 \mathrm{X}^{2} \quad\left(\mathrm{~N}=\text { teneur en azote des aiguilles }(\%) \text { et } \mathrm{x}=\frac{\text { altitude }}{1000}\right)
$$

On explique ainsi $28 \%$ de la variation de la nutrition azotée par l'altitude. 

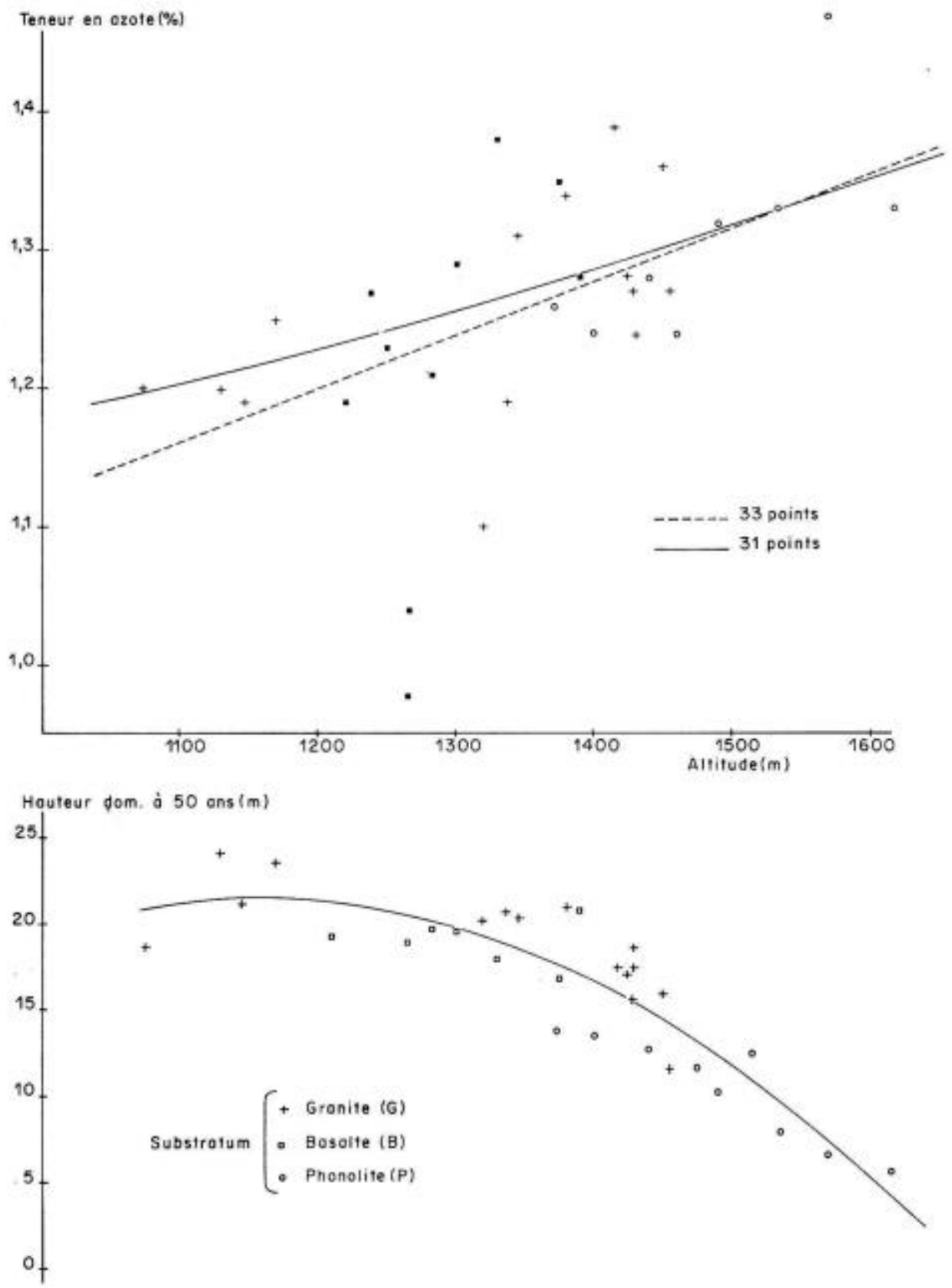

F10. 2. - Ajustement de la teneur en azote des aiguilles et de la hauteur dominante à 50 ans en fonction de l'altitude avec représentation des roches-mères

Fic. 2. - Relations between nitrogen content in needles and dominant height at age 50 with altitude 
TABLEAU 6 - TABLE 6

Coefficients de corrélation entre les teneurs en éléments minéraux des aiguilles et de l'altitude (ddl $=31 ; \mathrm{r}=0,349$ au seuil de $5 \%$ )

Correlation coefficients berween mineral composition of needles and allitude (ddl $=31: \mathrm{r}=0,349$ at the $5 \mathrm{So}$ level of significance)

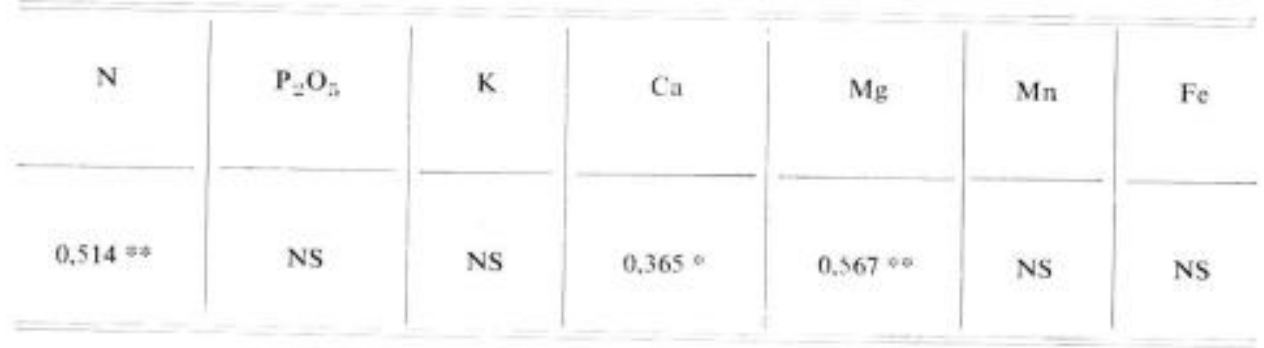

\section{V. - LIAISONS ENTRE NUTRITION ET PRODUCTION}

Nous avons essayé de déterminer dans quelle mesure les caractéristiques de nutrition pouvaient \& expliquer * les variations de production ou les \& composantes * de cette production. Nous avons utilis $\$$ comme variables à expliquer : la production totale (volume sur pieds et volume des éclaircies récupérées sur souches), la surface terrière, la circonférence dominante et la circonférence moyenne, la hauteur dominante et la hauteur moyenne, la hauteur dominante à 25 ans, la hauteur dominante à 50 ans, la longueur moyenne des aiguilles de l'année et le poids moyen de ces mêmes aiguilles.

Nous avons toujours procédé de la même manière : analyse des composantes principales sur les variables explicatives (caractéristiques de nutrition et âge), régression orthogonale et régression multiple à partir des variables explicatives initiales. Nous avons effectuée les calculs sur les logarithmes des variables en raison de l'allure non linéaire des liaisons (voir figure 2).

Disons immédiatement que les * explications * que nous obtenons sont loin d'être bonnes. Nous n'entrerons done pas dans les détails de toutes les analyses mathématiques que nous avons effectuées. Nous nous contenterons de donner un tableau des coefficients de corrélation totale et des coefficients de régression partielle significatifs (tableau 7) entre hauteur ou volume produit et les caractéristiques de nutrition. Nous donnerons également un tableau résumant les résultats de l'analyse par régression orthogonale effectuée sur la hauteur dominante à 50 ans (tableau 8). Ces tableaux résumənt assez bien les résultats. Nous voyons immédiatement que seuls le calcium et le magnésium présentent des liaisons constantes avec la production. Le calcium apparaît toujours significativement lié à la production lorsque l'on se borne au calcul des coefficients de corrélation totaux. Par contre, il n'apparaît plus dans les régressions multiples. Le magnésium au contraire, s'il apparait toujours dans les calculs de corrélation totale de manière très significative, reste presque toujours significatif dans les régressions multiples. 
Corrélation enire caractéristiques de nuirition et caractếrisitquess des peuplements

(Les coefficients de regrcssion partielle sont les coefficients bêta obtenus après lanalyse des composantes principalts lorsque lon repasse aux variables de départ)

Correlations berween mineral comrosition of needles and characteristics of Norway spruce stands (the partial regression costficients are the beta coefficienis obtained after the principal component analysis when we come back to the initial variables)

\begin{tabular}{|c|c|c|c|c|c|c|c|c|}
\hline $\begin{array}{l}\text { Eléments minéraux } \\
\text { des aiguilles }\end{array}$ & & $\mathrm{N}$ & $\mathrm{P}_{2} \mathrm{O}_{3}$ & $\mathrm{~K}$ & $\mathrm{Ca}$ & $\mathrm{Mg}$ & $\mathrm{Mn}$ & $\mathrm{Fe}$ \\
\hline \multirow{2}{*}{ Hauteur dominante ..... } & $\begin{array}{l}\text { cosfficient de corrélation } \\
\text { totale. }\end{array}$ & NS & NS & NS & $+0,35=$ & $+0,67=$ & NS & NS \\
\hline & $\begin{array}{c}\text { coafficient de régression } \\
\text { partielle. }\end{array}$ & NS & NS & NS & NS & $+0,437^{\circ}$ & NS & NS \\
\hline \multirow{2}{*}{ Hauteur moyenne ...... } & $\begin{array}{l}\text { coefficient de corrélation } \\
\text { totale. }\end{array}$ & NS & NS & NS & NS & $0,63=0$ & NS & NS \\
\hline & $\begin{array}{l}\text { coefficient de régression } \\
\text { partielle. }\end{array}$ & NS & NS & NS & NS & $+0,42=$ & NS & NS \\
\hline \multirow{2}{*}{$\begin{array}{r}\text { Hauteur } \\
25 \text { ans } \ldots \ldots \ldots \ldots \ldots . . .\end{array}$} & $\begin{array}{l}\text { coefficient de corrélation } \\
\text { totale. }\end{array}$ & $-0,39$ & NS & NS & $+0,52$ की & $+0,62$ हा & NS & NS \\
\hline & $\begin{array}{l}\text { cosfficient de régression } \\
\text { particlle. }\end{array}$ & NS & NS & NS & NS & NS & NS & NS \\
\hline \multirow{2}{*}{$\begin{array}{l}\text { Hauteur } \quad \text { dominante à } \\
50 \text { ans } \ldots \ldots \ldots \ldots \ldots \ldots\end{array}$} & $\begin{array}{l}\text { coefficient de correlation } \\
\text { totale. }\end{array}$ & $-0,37^{\circ}$ & NS & NS & $+0,51$ & $+0,64 \Rightarrow$ & NS & NS \\
\hline & $\begin{array}{l}\text { cosfficient de régression } \\
\text { partielle. }\end{array}$ & NS & NS & NS & NS & $+0,367^{\circ}$ & NS & NS \\
\hline \multirow{2}{*}{ Volume total.......} & $\begin{array}{l}\text { coefficient de corrélation } \\
\text { totale. }\end{array}$ & NS & NS & NS & NS & $+0,56$ & NS & NS \\
\hline & $\begin{array}{l}\text { coafficient de régression } \\
\text { partielle. }\end{array}$ & NS & NS & NS & Ns & $+0,48=$ & NS & NS \\
\hline
\end{tabular}


TABLEAU 8 - TABLE 8

Explicasion de la hauteur dominante à 50 ans en fonction de la nutrition par analyse de régression orthogonale

Explication of stand dominant height at age 50 in relation to mineral composition of needles by orthogonal regression

\begin{tabular}{|c|c|c|c|c|c|}
\hline $\begin{array}{c}\text { Compo- } \\
\text { sante- }\end{array}$ & $\begin{array}{l}\text { Valeur } \\
\text { propre }\end{array}$ & $\begin{array}{l}\text { Pourcen- } \\
\text { tage } \\
\text { cumulé }\end{array}$ & $\begin{array}{l}\text { Variables initiaies } \\
\text { intervenant dans la } \\
\text { composante }\end{array}$ & $\begin{array}{c}\text { Cocfficient de } \\
\text { corrélation avee } \\
\text { Ho50 ans }\end{array}$ & $\begin{array}{l}\text { Test de } \\
\text { Student }\end{array}$ \\
\hline 1 & 1,93 & 0,25 & $\begin{array}{c}+\log \mathrm{Ca}+\log \mathrm{Mg} \\
-\log \mathrm{N}\end{array}$ & $+0,574$ & $+4,18 \neq 0$ \\
\hline 2 & 1,81 & 0,47 & $\begin{array}{c}+\log \mathrm{P}_{. . \mathrm{O}_{5}}+\log \mathrm{K} \\
-\log \mathrm{Mn}\end{array}$ & $-0,302$ & $-2,20$ 하 \\
\hline
\end{tabular}

Si le calcium et le magnésium apparaissent liés à la production, en fait, ils ne doivent guère contribuer à l'explication des variations de production. En effet, ce sont les roches-mères les plus désaturées donc les plus pauvres en calcium et magnésium qui se trouvent aux altitudes les plus élevées et vice versa. Nous nous trouvons done en présence de deux phénomènes qui varient dans le même sens et qui sont corrélés entre eux essentiellement parce qu'ils dépendent d'un troisième facteur, l'altitude.

Il n’apparaît aucune liaison positive significative entre nutrition azotée et production, ce que laissaient prévoir les deux analyses de la variance et l'analyse canonique que nous avions précédemment effectuées.

Rappelons que BONNEAU (1967) a démontré d'une part que la prođuction d'azote minérale in situ est faible à haute altitude dans ce type de station, alors qu'elle devient élevée (en particulier la nitrification) à altitude plus basse, et que d'autre part, une amélioration de la disponibilité en calcium améliore considérablement la minéralisation de lazote. De même, Wıттіch (1961) a montré que la disponibilité en calcitum du sol avait uae importance considérable sur la production d'azote minérale. Or, dans la région où nous avons travaillé ces deux facteurs devraient se conjuguer pour entrainer une nette amélioration de la production azotée et done de la nutrition de lépicéa en cet élément à basse altitude.

Les analyses foliaires ne traduisent aucunement une amélioration de la nutrition azotée sur les stations les plus basses done les plus productives, et les plus riches en bases échangeables.

Il apparaît même une corrélation totale négative légèrement significative entre hauteur dominante à 25 ans ou à 50 ans et nutrition azotée. D'autre part, dans la régression orthogonale (tableau 8), une composante faisant intervenir négativement I'azote et positivement 'e calcium et le magnésium apparaît liée avec la hauteur dominante à 50 ans. Il semble done bien qu'il existe une liaison négative très légèrement significative entre production et nutrition azotée.

Nous allons tenter de donner une explication à cet état de fait. Une première chose est certaine: au niveau de nutrition auquel se trouvent les peuplements d'épicéa 
de Haute-Ardèche, il est impensable qu'une amélioration de la nutrition azotée puisse réellement entraîner une baisse de production.

Nous devons songer à mettre en cause le phénomène de dilution des éléments minéraux dans la masse foliaire.

Quelle que soit l'altitude, le niveau de nutrition azotée de J'épicéa est insuffisant. Mais nous pouvons admettre à la suite des travaux de BONNEAU qu'en réalité le potentiel de production azotée du sol est moins mauvais à basse altitude qu'à haute altitude.

Quand l'altitude augmente, le potentiel de production azotée du sol diminue, en raison principalement de labaissement de la température moyenne, de la diminution de la période d'activité des miero-organismes et de la désaturation du sol. De même, la production de l'épicéa diminue, mais dans des proportions plus considérables, car il s'ajoute aux effets de l'altitude proprement dits, les effets mécaniques du vent et de la neige qui finissent par annuler complètement la croissance.

La production de matière sèche diminue donc plus vite que la production azotée du sol et la teneur en azote des aiguilles a done tendance à augmenter. L'analyse foliaire ne peut done nous permettre de déceler les variations réelles des disponibilités en azote du milieu et traduit en fait l'inverse de ce qui se passe en réalité. Nous sommes en présence d'un cas où ce moyen d'investigation est pris en défaut. De nombreux auteurs ont souligné ces possibilités d'erreur d'interprétation en raison de ce phénomène de dilution des éléments minéraux dans la masse foliaire. Ici d'ailleurs, il est préférable de parler de phénomène de concentration en éléments minéraux par diminution de la masse foliaire, plutôt que de phénomène de dilution.

Nous pouvons maintenant nous poser la question de savoir pourquoi nous n'observons pas ici le même phénomène de concentration pour les autres éléments minéraux. En ce qui concerne le caleium et le magnésium, nous pouvons avancer lexplication suivante: la variation de disponibilité en calcium et magnésium est grande entre les sols issus de basalte (andosols mésotrophes) et les sols issus de phonolite (andosols oligotrophes). La teneur en calcium échangeable peut descendre à $0.05 \mathrm{~m}$.e. pour $100 \mathrm{~g}$ sur phonolite et atteindre près de 30 m.e. pour $100 \mathrm{~g}$ sur basalte (LE TACoN, Oswald 1969). De même, le taux de saturation peut varier de $2 \%$ à près de $50 \%$.

Sur granite les variations ont une moins grande amplitude, mais sont encore importantes. A haute altitude dans les horizons A, le taux de saturation peut descendre à moins de $2 \%$ dans les rankerss issus des granites.

Nous pouvons penser que la diminution de disponibilité en $\mathrm{Ca}$ et $\mathrm{Mg}$ du sol n'est pas compens se entièrement par la diminution de la masse foliaire. Les phénomènes de concentration ne sont pas suffisants pour masquer complètement la diminution du niveau réel de la teneur ea calcium et magnésium des aiguilles.

Le niveau de nutrition en potassium et phosphore est toujours à un niveau élevé. La diminution de la masse foliaire ne peut done se traduire par une augmentation conséquente de la teneur en ces éléments dans les aiguilles.

Néanmoins, si nous étudions plus attentivement les liaisons pouvant exister entre hauteur dominante et nutrition par régression orthogonale (tableau 8), nous constatons que la composante $\mathrm{n}^{\prime \prime} 2$ est significativement liée, mais négativement, à la hau- 
teur dominante à 50 ans. Or, cette composante fait intervenir positivement le potassium et le phosphore, et négativement le manganèse. Il y a donc bien une faible liaison négative entre teneur en phosphore et potassium des aiguilles et production.

Nous pouvons raisonnablement interpréter cette faible liaison négative comme la conséquence du phénomène de concentration. Mais l'action de ce phénomène de concentration est à peine décelable en raison du niveau atteint par le phosphore et le potassium, niveau qui est maximal quelle que soit l'altitude, ce qui n'est évidemment pas le cas de l'azote.

Enfin, apparemment le phénomène de concentration induit par la diminution de la masse de matière sèche synthétisée ne se répercute pas sur la nutrition en manganèse et en fer en raison de la variation importante de ces deux éléments, et surtout du manganèse dans les aiguilles,

\section{VI. - LIAISONS ENTRE NUTRITION ET CARACTERISTIQUES DU SOL}

Nous allons essayer de voir dans quelle mesure les caractéristiques de nutrition peuvent être sexpliquées s par les caractéristiques du sol. Nous avons procédé systématiquement horizon par horizon en utilisant comme précédemment l'analyse par régression orthogonale et l'analyse par régression multiple.

Il ne fallait pas s'attendre à trouver de bonnes explications pour diverses raisons. Tout d'abord, nous avons vu qu'un phénomène sparasite * leffet de concentration, est venu complètement perturber la teneur en éléments minéraux des aiguilles.

Le potassium et le phosphore sont toujours à un niveau élevé dans les aiguilles. Il est done vain de rechercher des liaisons entre phosphore du sol et phosphore des aiguilles, ou potassium du sol et potassium des aiguilles. En ce qui concerne lazote, l'effet de concentration a inversé la variation dans les aiguilles et d'autre part, l'azote total que nous avons dosé dans le sol est bien insuffisant pour nous donner une idée de la possibilité réelle de fourniture d'azote minéral.

II reste maintenant le cas du calcium, du magnésium et du manganèse. Nous avons déjà mis en évidence l'influence de la roche-mère sur la nutrition en calcium et magnésium. En utilisant toute l'information et en essayant d'interpréter plus finement les résultats, nous n'arrivons pas à une meilleure \& explication \$, très certainement en raison de l'influence perturbatrice de l'effet de concentration.

Pour le manganèse, nous avons obtenu des résultats beaucoup plus spectaculaires, car sa variation est très importante dans les aiguilles (test $F$ très élevé dans l'analyse de la variance) et, par conséquent, a été peu influencée, comme nous l'avons déjà souligné, par l'effet de concentration.

Ce sont les caractéristiques de Ihorizon $A_{1}$ qui nous donnent la meilleure explication des variations de la teneur en manganèse des aiguilles: horizon $A_{0}: R^{2}=0.74$, horizon $\mathrm{A}_{1}: \mathrm{R}^{2}=0,87$, horizon $\mathrm{B}-\mathrm{C}: \mathrm{R}^{2}=0,81$. L'explication est toujours élevée quels que soient les horizons, et atteint près de $90 \%$ avec les horizons $A_{1}$. Ce résultat est tout à fait remarquable, car il a été obtenu sans que nous utilisions les teneurs en manganèse du sol.

Nous allons maintenant commenter ces résultats en n'utilisant que les caractéristiques des horizons $\mathbf{A}_{1}$. 


\section{TABleav 9 - TABle 9}

Matrice des coefficients de corrétations totanx entre $\mathrm{Mn}$ (aivuilles) et caractérisfiques des horizons $A_{1}$. Les valeurs significatives sont soulignées en pointillé pour $P=0,05$ et en trait plein pour

Matrix of total correlation coefficients between $M n$ of nzedles and characteristics of $A$, horizons.

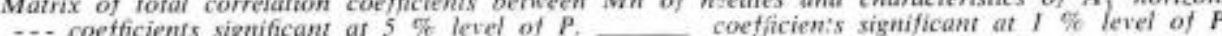

\begin{tabular}{|c|c|c|c|c|c|c|c|c|c|c|c|c|c|c|c|}
\hline Arg $\quad \ldots . .$. & 1,00 & & & & & & & & & & & & & & \\
\hline Lf $\ldots \ldots \ldots$ & 0,78 & 1,00 & & & & & & & & & & & & & \\
\hline $\mathrm{Lg}, \ldots \ldots \ldots$ & 0.67 & 0.92 & 1,00 & & & & & & & & & & & & \\
\hline si $\quad \ldots . . .$. & -0.27 & $-0,32$ & -0.09 & 1,00 & & & & & & & & & & & \\
\hline $\mathrm{Sg} \ldots \ldots \ldots$ & $-0,67$ & $-0,84$ & $-0,71$ & 0,70 & 1,00 & & & & & & & & & & \\
\hline $\mathbf{N}$ total .... & 0,70 & 0,63 & 0,43 & -0.58 & -0.75 & 1,00 & & & & & & & & & \\
\hline $\mathrm{pH} \quad \ldots . .$. & 0,07 & 0.26 & 0,22 & 0,21 & 0.05 & -0.07 & 1.00 & & & & & & & & \\
\hline $\mathrm{Ca}++\ldots$. & 0.10 & 0,05 & $-0,08$ & 0,21 & 0.14 & 0,12 & 0.63 & 1,00 & & & & & & & \\
\hline $\mathrm{K}+\ldots \ldots \ldots$ & 0,20 & $-0,04$ & $-0,22$ & $-0,38$ & -0.11 & 0,47 & -0.19 & -0.25 & 1,00 & & & & & & \\
\hline $\mathrm{Mg}++\ldots$. & 0,44 & 0,47 & 0,36 & 0.15 & -0.18 & 0,22 & 0.76 & 0,71 & $-0,01$ & 1,00 & & & & & \\
\hline $\mathbf{T} \ldots \ldots \ldots$ & 0,69 & 0,66 & 0,47 & $-0,55$ & -0.76 & 0.94 & 001 & 0,16 & 0,40 & 0,30 & 1,00 & & & & \\
\hline $\mathrm{P}_{3} \mathrm{O}_{5} \quad \ldots \ldots$ & 0,05 & 0,27 & 0,32 & 0.31 & -0.00 & -0.07 & 0,70 & 0,45 & $-0,22$ & 0,53 & 0,02 & 1,00 & & & \\
\hline Epaisseur $A_{1}$ & 0,13 & 0,29 & 0,32 & -0.04 & $-0,30$ & 0,12 & 0,41 & 0.15 & $-0,08$ & 0,28 & 0,19 & 0,21 & 1.09 & & \\
\hline $\mathrm{C} / \mathrm{N} \quad \ldots \ldots$ & $-0,56$ & $-0,56$ & -0.59 & -0.06 & 0,38 & $-0,28$ & -0.55 & -0.27 & 0,06 & $-0,52$ & 0,23 & 0.52 & $-0,31$ & 1,03 & \\
\hline $\mathrm{S} / \mathrm{T} \ldots \ldots$. & 0,01 & 0,02 & $-0,07$ & 0,33 & 0,24 & $-0,09$ & 0.73 & 0,91 & 0,12 & 0,79 & $-0,04$ & $-0,61$ & 0,11 & $-0,30$ & 1,00 \\
\hline Mn aiguilles & $-0,41$ & -0.50 & $-0,42$ & 0,04 & 0,35 & $-0,23$ & -0.74 & -0.62 & -0.16 & $-0,68$ & $-0,34$ & -0.61 & -0.52 & -0.67 & $-0,57$ \\
\hline & Arg & L.g & L.g & $-\mathrm{Sf}$ & $-\mathrm{Sg}$ & $\mathrm{N}$ total & $\mathrm{pH}$ & $\mathrm{Ca}$ & $\mathrm{K}$ & $\mathrm{Mg}$ & $\mathrm{T}$ & $\mathrm{P}_{2} \mathrm{O}_{5}$ & $\begin{array}{l}\text { Epais- } \\
\text { ssur Al }\end{array}$ & $\mathrm{C} / \mathrm{N}$ & S $/ \mathbf{T}$ \\
\hline
\end{tabular}




\section{TABLEAU 10 - TABLE 10}

Explication de la teneur en $M n$ des aiguilles en fonction des caractéristiques des horizons $A_{1}$ par analyse de régression orihogonale

Explication of the level of manganese mutrition in relation to the characteristics of $A_{1}$ horizons by orthogonal regression

\begin{tabular}{|c|c|c|c|c|c|}
\hline Composante & $\begin{array}{l}\text { Valeur } \\
\text { propre }\end{array}$ & $\begin{array}{l}\text { Pourcentage } \\
\text { d'explication } \\
\text { cumule }\end{array}$ & $\begin{array}{l}\text { Variables initiales intervenant } \\
\text { dans la composante }\end{array}$ & $\begin{array}{l}\text { Coefficient de } \\
\text { corrélation avec } \\
\text { Mn aiguilles }\end{array}$ & Test de Student \\
\hline 1 & 5,64 & 0,57 & $\begin{array}{c}+\log \text { Argile }+\log \text { L.f }+\log \text { L.g } \\
-\log S+\log T\end{array}$ & $-0,71$ & $-8,21=0$ \\
\hline 2 & 4,05 & 0,64 & 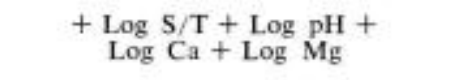 & $-0,46$ & $-5,34=7$ \\
\hline 3 & 1,98 & 0,78 & $+\log K$ & $-0,097$ & NS \\
\hline 4 & 0,86 & 0,84 & Log épaisseur $\mathrm{A}_{1}$ & $-0,22$ & $4,58 *$ \\
\hline
\end{tabular}


La matrice des coefficients de corrélation totale nous donne déjæ̀ des résultats assez clairs (tableau 9). La teneur en manganèse des aiguilles est très liéc d'une part avec la granulométrie et, d'autre part, avec la saturation du complexe absorbant et les variables qui y sont liées ( $\mathrm{S} / \mathrm{T}, \mathrm{pH}, \mathrm{Ca}$ échangeable et $\mathrm{M}_{\mathrm{g}}$ échangeable).

De plus, les coefficients de corrélation totaux sont significatifs entre $\mathrm{Mn}$ des aiguilles et épaisseur de l'horizon $\mathrm{A}_{1}$ teneur en phosphore et $\mathrm{C} / \mathrm{N}$.

Ces résultats s'améliorent très sensiblement par régression orthogonale (tabieau 10). Si nous ne gardons que les quatre premières compozantes qui, à elles seules, expliquent $84 \%$ de la variation, nous obtenons tout d'abord un \& regroupement * excellent des caractéristiques du sol.

La première composante ne fait intervenir que la granulométrie et la capacité totale d'échange qui en dépend fortement. Les éléments fins interviennent positivement ainsi que la capacité totale d'échange alors que les sables grossiers interviennent négativement. Cette composante est très liée (coefficient de corrélation de 0,71 ) avec la teneur en manganèse des aiguilles, mais négativement.

La deuxième composante fait intervenir le taux de saturation, le $\mathrm{pH}$ et la teneur en calcium et magnésium échangeables. Elle est également très liée négativement avec la teneur en manganèse des aiguilles.

La troisième composante qui ne fait intervenir que le potassium échangeable n'est absolument pas liée avec la nutrition en manganèse. Enfin, la quatrième composante qui ne fait intervenir que l'épaisseur de l'horizon $A_{1}$ est légèrement liée négativement avec la nutrition en manganèse.

Si la granulométrie apparait très liée à la nutrition en manganèse, cette liaison n'est probablement pas une véritable liaison de cause à effet ; elle provient vraisemblablement du fait que la disponibilité du sol en manganèse est plus élevée sur granite que sur les roches effusives. Or, les sols issus du granite ont une texture grossière et les sols issus de roches effusives, une texture fine.

La véritable liaison fonctionnelle est très certainement traduite par l'influence de la deuxième composante : taux de saturation, $\mathrm{pH}$. $\mathrm{Ca}$ et $\mathrm{Mg}$ échangeables. $\mathrm{Ce}$ sont d'ailleurs du calcium et du magnésium échangeables que dépendent le $\mathrm{pH}$ et le taux de saturation, et ce sont, en fait, de ces deux éléments que dépend véritablement le plus, le niveau de nutrition en manganèse.

Dans une précédente étude (LE TACON, MLLlier 1970) nous avions déjà montré l'influence considérable de la teneur en calcium du sol sur la nutrition en manganèse de l'épicéa. Nous avions supposé que le calcium du sol agissait plus en bloquant l'absorption du manganèse qu'en provoquant la rétrogradation du manganèse sous forme inassimilable. Les résultats obtenus ici avee des sols dont le $\mathrm{pH}$ (horizon $\mathrm{A}_{1}$ ) ne dépasse pas 5,5 confirment cette façon de voir et de plus, montrent que le magnésium joue un rôle identique à celui du calcium.

Les rôles très voisins joués par le magnésium et le calcium sont particulièrement bien mis en évidence par une analyse des correspondances sur l'ensemble des 33 placettes et sur les 39 analyses de sol des trois horizons (13 analyses par horizon). Cette méthode nouvelle d'analyse des données a été imaginée et mise au point par BENZECRI J.-P. (1969): voisine de l'analyse des composantes principales, elle s'attache à l'étude des \&profils ? de variables plus qu’à leur valeur absolue ; en outre elle fait 
jouer un rôle tout à fait symétrique aux placettes et aux variables. Plus des $4 / 5$ de la variabilité entre les 39 variables sont résumés dans le plan de la figure 3 ; l'analyse isole de façon excellente le rôle très particulier du calcium et du magnésium dont les points représentatifs des trois horizons sont regroupés dans le coin gauche en bas du graphique ; elle isole aussi les sables des différents horizons.

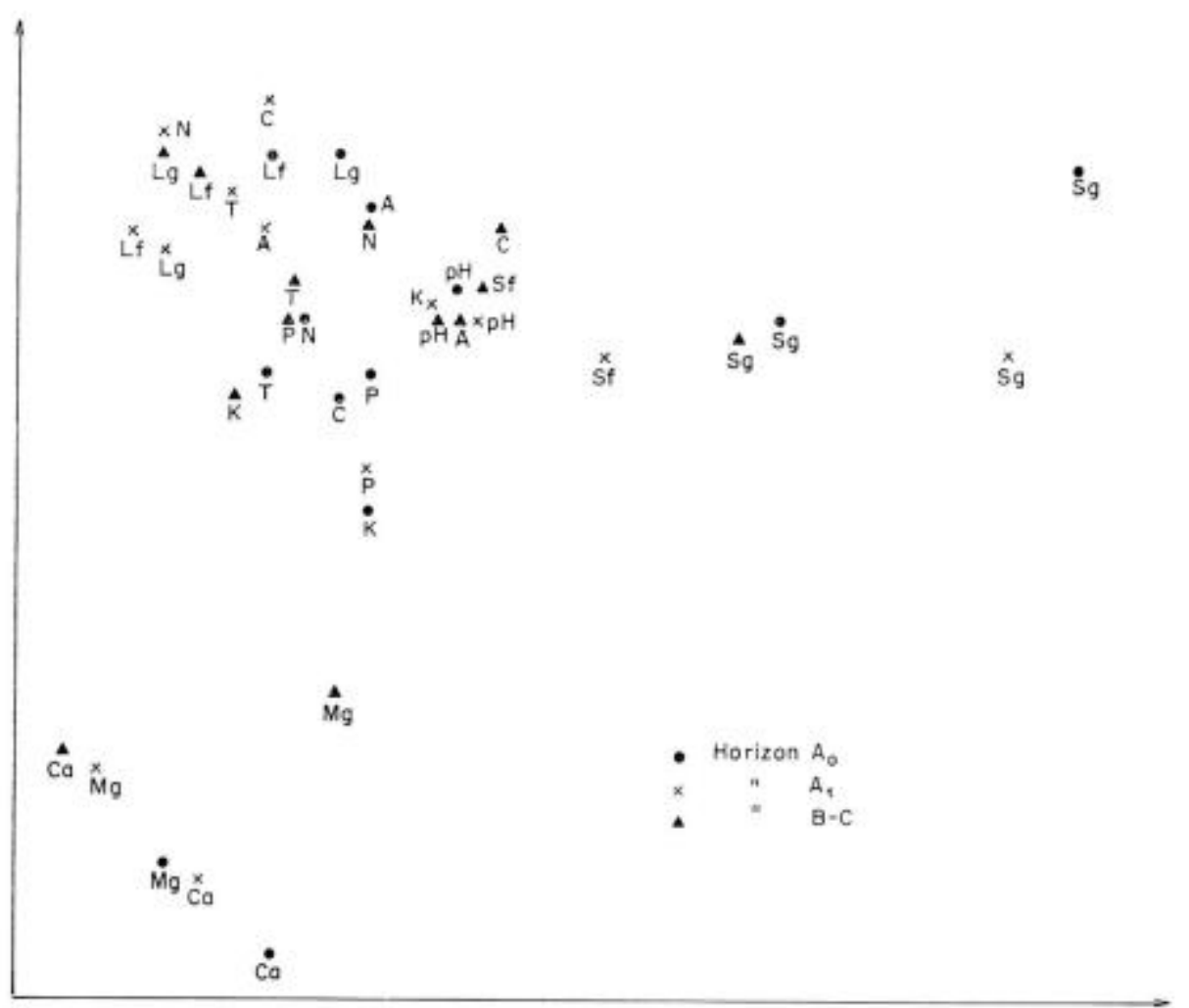

Fic. 3. - Représentation graphique des rèsultats de Tanalyse des correspondances pour les 39 variables des horizons $A_{i}$ et $A_{1}$ et $(B)-C$, dans le plan $F_{1}-F_{2}$

FiG. 3. - Graph of results of "correspondence analysis s for the 39 variables of $A o, A_{1}$ and $(B)$ $C$ horizons, in the plane of $F_{I}-F_{A}$

\section{CONCLUSIONS}

La production apparaît liée positivement à la teneur en calcium et magnésium des aiguilles et négativement à la teneur en azote, phosphore et potassium des aiguilles (liaison très légèrement significative en ce qui concerne le phosphore et le potassium).

Ces résultats demandent à être interprétés avec beaucoup de prudence car les liaisons ainsi mises en évidence ne sont nullement des liaisons de cause à effet. La liaison positive entre production et nutrition en calcium et magnésium nous semble simplement provenir du fait que la production diminue avec l'altitude et que les sols les plus pauvres en bases échangeables sont situés à haute altitude. 
Les liaisons négatives entre production et nutrition en azote, phosphore et potassium ne traduisent pas non plus des liaisons de cause à effet, mais un phénomène de dilution ou plus exactement de concentration des éléments minéraux dans la masse foliaire. Quand l'altitude augmente, sous l'effet de la diminution de température, de la diminution de la période de végétation et surtout sous l'action du vent et de la neige la masse de matière sèche produite diminue considérablement. Cette diminution de la biomasse a tendance à provoquer une augmentation de la teneur en azote des aiguilles et, à un degré moindre, de la teneur en phosphore et en potassium, car ces đéux éléments sont toujours à leur concentration maximale. En réalité, les variations de production ne sont pratiquement pas explicables par les variations de nutrition. C'est Taltitude seule qui, par se: effets propres, est directement responsable de la chute de production.

Nous sommes en présence d'un cas où l'investigation par analyse foliaire est prise en partie en défaut et où les résultats des différentes techniques statistiques doivent être interprétés avec beaucoup de prudence car ils mettent en évidence des liaisons qui, ici, ne sont jamais des liaisons causales.

Par contre, la teneur en manganèse des aiguilles qui présente une grande variabilité r’a guère été affectée, contrairement à la teneur des autres éléments, par le phénomène de concentration.

Nous avons ainsi obtenu une excellente explication * de la variabilité de la teneur en manganèse des aiguilles par les caractéristiques du sol et essentiellement par les caractéristiques des horizons $A_{1}$.

La variation du niveau de nutrition en manganèse dépend fortement de la disponibilité en calcium et magnésium échangeables du sol, ce qui rejoint des résultats analogues obtent's dans l'est de la France, où nous avions émi; Thypothèse de l'existence d'un phénomène d'antagonisme entre calcium et manganèse, indépendamment de tout phénomène de rétrogradation dans le sol.

Le manganèse est l'élément qui discrimine le plus, et de très loin. l'ensemble des peuplements, alors que l'azote est l'élément le moins discriminant.

La nutrition de l'épicéa sur les trois roches-mères, phonolite, granite et basalte, diffère par trois éléments surtout, le calcium, le manganèse et le magnésium et à un degré bien moindre par un quatrième, le phosphore.

Globalement, la nutrition de l'épicéa en Haute-Ardèche est à un niveau très satisfaisant pour tous les éléments, sauf pour l'azote qui présente systématiquement des déficiences marquées. Même à l'altitude la plus basse où nous avons travaillé, c'est-àdire à $1.000 \mathrm{~m}$ environ et même sur des roches-mères bien pourvues en base échangeable, comme les basaltes, le niveau de nutrition azotée est très insuffisant. Les peuplements situés sur basaltes ont un niveau moyen en azote dans les aiguilles de 1,22\% seulement, ce qui est très étonnant. Sur les autres roches-mères, le niveau de nutrition est d'ailleurs tout aussi mauvais, mais celà est moins étonnant en raison de leur plus faible teneur en calcium.

Avec des teneurs aussi basses en azote, la production varie néanmoins entre 10.5 et $12 \mathrm{~m}^{3}$ par hectare et par an à 75 ans entre 1.000 et $1.350 \mathrm{~m}$ d'altitude. Nous pou- 
vons espérer, par une fertilisation azotée, améliorer considérablement la production de bois. Différents essais de fertilisation ont été mis en place ou vont être mis en place sur des peuplements d'âge compris entre 60 et 80 ans. En plus de l'azote, nous testerons également l'effet du calcium sur les roches-mères acides (phonolite et granite). C'est probablement dans ces types de station de moyenne altitude du Massif Central que la fertilisation des peuplements adultes autorise les plus grands espoirs. En effet, un seul élément est déficitaire, ce qui réduira le coût des interventions, le potentiel de production est très important et, de plus, le prix du $\mathrm{m}^{*}$ de bois d'épicéa est très élevé.

Resu pour publication en octobre 1970.

\section{SUMMARY}

\section{RELATIONS BETWEEN YIELD AND MINERAL NUTRITION OF NORWAY SPRUCE IN} \& HAUTE-ARDÈCHE *

A positive relation seems to exist between Norway-spruce yield and calcium and magnesium contents of needles, and a negative one between yield and nitrogen, phosphorus and potassium zontents of needles. This relation seems to be of little significance as far as phosphorus and potassium are concerned.

These relations however, dot not at all seem to be cause and effect relations. The positive iclation between yield and calcium and magnesium nutrition seems to stem simply from the fact that yield decreases with altitude and that the soils are poorest in exchangeable bases at high altitude.

Neither do negative relations between yield and nitrogen, phosphorus and potassium nutrition reflect cause and effect relations, but a phenomenon of concentration of mineral elements in needies. When altitude increases the quantity of dry matter synthesized diminisles considerably due to lower temperature, shorter vegetation period and above all due to the action of wind and snow. This reduction of foliage weight tends to provoke an increase of the nitrogen content in needles. The increase of phosphorus and potassium contents in needles is less significant, since the content of these two elements is always at a high level.

In reality, the variations of yield cannot be explained by variations of nutrition. It is altitude alone, which causes by its inherent effects the decrease of production.

On the other hand, manganese content in needles, which shows great variability is hardly affected by phenomenons of concentration.

We found that soil characteristics, essentially characteristics of $A_{1}$ horizens, offer an excellent explanation $>$ of variability of manganese content in needles.

Tre level of manganese nutrition depends on availability of exchangeable calcium and magnesium in soil, which confirms similar results obtained in eastern France (LE TACON, MLLLIER, 1970).

Manganese is the most discriminant element by far for the totality of Norway spruce stands, whereas nitrogen is the least discriminant one.

Fssentially three elements : calcium, manganese and magnesium (and to a lesser degree phosphorus as well) differentiate nutrition of Norway spruce on the three parent-rocks: phonolite, granite and basalt.

Nutrition of Norway spruce in Haute-Ardèche is at a very satisfactory level for all elements, except for nitrogen, the level of which always shows marked deficiencies. At altitude of about $1000 \mathrm{~m}$ and even on parent-rocks rich in exchangeable calcium (basalts e.g.) nitiogen nutrition level is extremely insufficient.

Average nitrogen content of needles is 1.22 p.c. only on basalt.

Nutriticn level is as bad on other parent-rocks. Due to their lower exchangeable calcium content, this is, however, less astonishing. 
Even with as low nitrogen contents, mean annual volume increment at age 75 fluctuates between 10.5 and $12 \mathrm{~m}^{3}$ per hectare, between 1000 and $1350 \mathrm{~m}$ altitude. We may hope to increase yield considerably by nitrogen fertilization. Several fortilization experiments are being conducted, or will be conducted on Norway sptuce stands between 60 and 80 years old.

\title{
ZUSAMMENFASSUNG
}

\section{DIE MINERALSTOFFERNAHRUNG DER FICHTE IM \& HAUTE-ARDÈCHE $*$ :}

\author{
ERNÄHRUNGZUSTAND UND WUCHSLEISTUNG
}

In der vorliegenden Arbeit wird der Ernihhrungszustand der Fichte auf den Hochplateaus der Departments Ardèche und Haute-Loire im französischen Zentralmassiv untersucht. Eine Wuchsleistungsanalyse, in Abhängigkeit von verschiedenen Standortsmerkmalen wie Seehöhe, Grurdgestein, Wind, Exposition und Hangneigung (OswALD, 1969) und eine eingehende Beschreibung der Böden (LE TACON, OSWALD, 1969) aus diesem Gebiet wurde bereits veröffentlicht, sodass hier nicht näher darauf eingegangen wurde.

In Tabelle I sind die Ergebnisse der Nadelanalysen sowie die wesentlichsten Standorts und Bestandesmerkmale der 33 Versuchsflächen wiedergegeben. Man ersieht daraus, dass mit Ausnahme von Stickstoff alle übrigen Elemente in ausreichender Menge vorhanden sind, oder zumindest über dem Karrenzniveau liegen. Auf allen Standorten konnte ein deutlicher Stickstoffmangel verzeichnet werden und zwar auch auf ausreichend mit Basen versorgten Böden auf Basalt im unteren Teil unseres Arbeitsgebietes d.h. zwischen 1.000 und $1.200 \mathrm{~m}$ Seehöhe. Trotz der geringen Stickstoffwerte in den Nadeln haben wir zwischen 1.000 und $1.350 \mathrm{~m}$ Sechöbe noch einen DGZ von $10 \mathrm{~b}: \mathrm{s} 12 \mathrm{fm}$ im Alter $75 \mathrm{zu}$ verzeichnen. Man kann daher hoffen, dass eine alleinige Stickstoffdüngung in Beständen zwischen 60 und 80 Jahren noch einen beträchtlichen Zuwachsgewinn erbringen kann. Ve suche in dieser Richtung wurden bereits angelegt, wobei zusitzlich eine Kalziumgabe auf siurem Grundgestein (Granite, Phonolith) auf ihre Wirkung untersucht werden soll.

Eine einfache Streuungszerlegung zeigt, dass bei allen Elementen insbesondere bei Mangan. Unterschiede zwischen den Beständen bestehen.

Der Ernährungszustand der Bestainde auf den drei Gundgesteinsgruppan unterscheidet sich durch eine Magnesiumkomponente und eine Kalzium- Mangankomponente; die beiden letzeren Elemente haben entgegengesetzte Vorzeichen (Fig. 1 A und fig. 1 B).

Im Abschnitt 5 werden die Zusammenhänge zwischen Ernährungszustand und Wuchsleistung (verschiedene Bestandesmerkmale) untersucht. Die in Tab. 7 zusammengefassten Ergebnisse müssen mit grosser Vorsicht interpretiert werden, da es sich hier keinesfalls um kausale Zusammenhänge handelt. Dies zeigt sich besonders deutlich bei der positiven Beziehung zwischen der Wuchsleistung und der Kalzium - und Magnesiumernährung welche von einem dritten Faktor, der Seehöhe, abhängt, wobei die basenreichen Basaltstandorte ausschliesslich im unteren Teil des Arbeitsgebietes vorkommen. Die negative Beziehung zwischen Oberhōhe im Alter 50 und Stickstoffernährung (Komponente 1. Tab. 8) sowie die Tendenz einer Zunahme des Nadelstickstoffgehaltes mit der Seehöte (Abb. 2) kann vielleicht als ein Verdünnungseffekt, oder besser als ein Konzentrationseffekt erklärt werden, der eng mit der Abnahme der Biomasse, insbesondere der Nadelmasse, mit steigender Seehöhe zusammenhängen dürfte. Die Interpretation der Nadelanalysenergebniss? stösst hier auf besondere Schwierigkeiten und muss mit grösster Vorsicht vorgenommen werden.

Im Abschnitt 6. wurden die Zuzammenhänge zwischen den Nährstoffspiegelwerten und den physikalischen und chemischen Bodenanalysenergebnissen untersucht, wobei $85 \%$ der Variation der Mangan - Nadelspiegelwerte mit den interpretierbaren Prinzipalkomponenten des $A_{1}$ Horizontes erklärt werden konnten (Tab. 9 u. Tab. 10).

Eine neue statistische Analysenmethode (analyse des correspondances) welche die 39 Variablen der drei Bodenhorizonte in den 33 Probeflächen zusammenfasst, bringt die spezielle Bedeutung von Kalzium und Magnesium deutlich zum Ausdruck (Abb. 3). 


\section{REFERENCES BIBLIOGRAPHIQUES}

Baule H., Frjeker C., 1969. La fertilisation des arbres forestiers, Munich, 255 pages,

BENzECRI J.-P., 1969. Leçons sar Tanalyse factorielle et la reconnaissance des formes, texte ronéotypé, I.S.U.P., Paris.

Bonneau M., 1962. Les sols d'altitude de l'étage subalpin et leur reboisement. Bull. A.F.E.S. (4) p. $216-223$.

Bonkeau M., 1965. Premiers résultats d'essais de fertilisation de l'Epicéa à haute altitude dans le Massif Central, Amn. Sci, forest, 12, 3, p. 357-393.

Bonneau M. 1967. Minéralisation de l'azote de deux so's des Hautes Vosgis, Allures du phénomène et tentatives d'amélioration. $S_{c i}$. sol., $\mathrm{n}^{\circ}$ 1, p. 19-48.

Corder, 1965. L'analyse factorielle des correspondances. These, 3 r cycle. Rennes, 66 p.

Duchaufour $\mathrm{Ph} ., 1957$. Un cas de carence azoté de l'Epicéa décelée par le diagnostic foliaire. Rev, forest, fr, n" 2.

FIEDER H. J., NeBe W., 1969. Ober Emährung und Höhenwachstum von Fichtenbeständen in den oberen Berg und Kammlagen des Osterzgebirges. Archiv. für Forstwesen, 18, 7, p. 747-756.

LE TACON F. OswaLd H., 1969. Les sols de Haute-Ardèche, caractérisation et production forestière. Bulletin A.F.E.S., n" 2, mars-avril 1969, p. 7 aे 17.

Le Tacon F., Milteier C., 1970. La nutrition minérale de l'épicéa commun en sol carbonaté et en sol dácarbonaté. Essai sur le comportement du calcium et du manganèse. Ann. Sci. forest., $n^{\circ} 1, p, 1$ à 24.

LundegardH H., 1945. Die Blattanalyse Gustav, Fischer. Verlag Jena.

Oswald H. 1969. Conditions forestières et potentialité de I'Epiécé en Haute-Ardèche. Ann. Sci. forest., 26, $\pi^{\circ} 2$, D. $183-224$.

Stefnajered $F_{n}$, 1954. Manuting, plant production and the chemical composition of thz plant. Plant and Soil, 5, p. 226-242.

STEENBJEk F., 1950. On the relative contents of plant nutrients in crops. Trans. 4. Intern. Congr. Soil. Sci, 1, 254-258.

Tomassone R., 1967. Une méthode d'investigation: la régression orthogonale. Ann. Sci. forest., 24, (3). p. $233-258$.

Wrтисн W. 1952. Der heutige Stand unseres Wissens vom Humus und neue Wege zur Läsung des Rohhumusproblems im Walde (Schriftenreihe der forst. Fakultät der. Univ. Göttingen), J. D. Sauerlander's Verlag. Frankfurt/Main, 4-106 p.

WurncH W. 1961. Bodenkundliche und planzenphysiologische Grundlagen der Kalkdïngung im Walde. Der Wald braucht Kalk $n^{\circ} 3$. 